\title{
Joint Parameter Estimation for UWB Energy Detectors Using OOK
}

\author{
Mustafa E. Şahin, İsmail Güvenç, and Hüseyin Arslan \\ Electrical Engineering Department, University of South Florida, Tampa, FL, United States \\ E-mail: $\{$ mesahin, iguvenc, arslan\}@eng.usf.edu
}

\begin{abstract}
In this paper, joint estimation of the optimal threshold, synchronization point, and integration interval is developed for ultrawideband (UWB) energy detectors employing on-off keying (OOK) modulation. Gaussian approximation of the received signal statistics is shown to enable low complexity solutions at the expense of some performance degradation. The performances of the optimal and suboptimal solutions are evaluated and compared. It is shown that explicit BER minimization is required for parameter optimization. Using simulations, number of training symbols required to converge to ideal parameter estimates is demonstrated to be increasing with increasing SNR.
\end{abstract}

\section{Index Terms}

ultrawideband, UWB, energy detector, threshold, optimum integration interval

\section{INTRODUCTION}

Energy detector is a non-coherent approach for ultrawideband (UWB) signal reception, where low complexity receivers can be achieved at the expense of some performance degradation [1]. As opposed to more complex RAKE receivers, estimation of individual pulse shapes, path amplitudes, and delays at each multipath component is not necessary for energy detectors. Moreover, energy detectors are less sensitive against synchronization errors [2], and are capable of collecting the energy from all the multipath components.

On-off keying (OOK) is one of the most popular non-coherent modulation options that has been considered for energy detectors. OOK based implementation of energy detectors is achieved by passing the signal through a square law device (such as a Schottky diode operating in square-region) followed by an integrator and a decision mechanism, where the decisions are made by comparing the outputs of the integrator with a threshold. Two challenging issues for the enhancement of energy detector receivers are the estimation of the optimal threshold, and the determination of synchronization/dump points of the integrator.

The effect of integration interval on the system performance has been analyzed before for energy detectors [1], [3]. However, to our best knowledge, optimal joint selection of the integration start and stop times, and the threshold is not covered in the literature. In this work, our contributions are as follows: 1- We address the optimal joint parameter selection using the bit error rate (BER) expressions with exact analysis and Gaussian approximation (GA), and show that GA works well only at large bandwidths, 2- We define a framework for synchronization/dump hypotheses with different sampling options, 3- When an exact analysis is considered, using the GA for calculating the threshold yields very small performance losses, and can be considered as a practical alternative for exact threshold evaluation, 4- The parameter estimation requires explicit BER minimization (rather than SNR maximization) since the statistics corresponding to different bits are not identical, 5- The number of training symbols required to converge to the ideal parameter estimates is shown to be less than one hundred for practical operating scenarios.

The paper is organized as follows. In Section II system model for an UWB system employing OOK is presented. Optimum selection of integration interval start/stop times, and the threshold is addressed in Section III, exact and Gaussian approximation methods for BER evaluation are analyzed in Section IV, and the numerical results are presented in Section V. 


\begin{tabular}{|c|c|c|c|c|}
\hline \multicolumn{2}{|c|}{$\begin{array}{l}\text { Parameter } \\
\text { Estimation }\end{array}$} & \multirow[t]{2}{*}{ Symbol Detection } & \\
\hline '0's & '1's & & '0's & '1's \\
\hline
\end{tabular}

Fig. 1. Parameter estimation and symbol detection in block-fading channel model

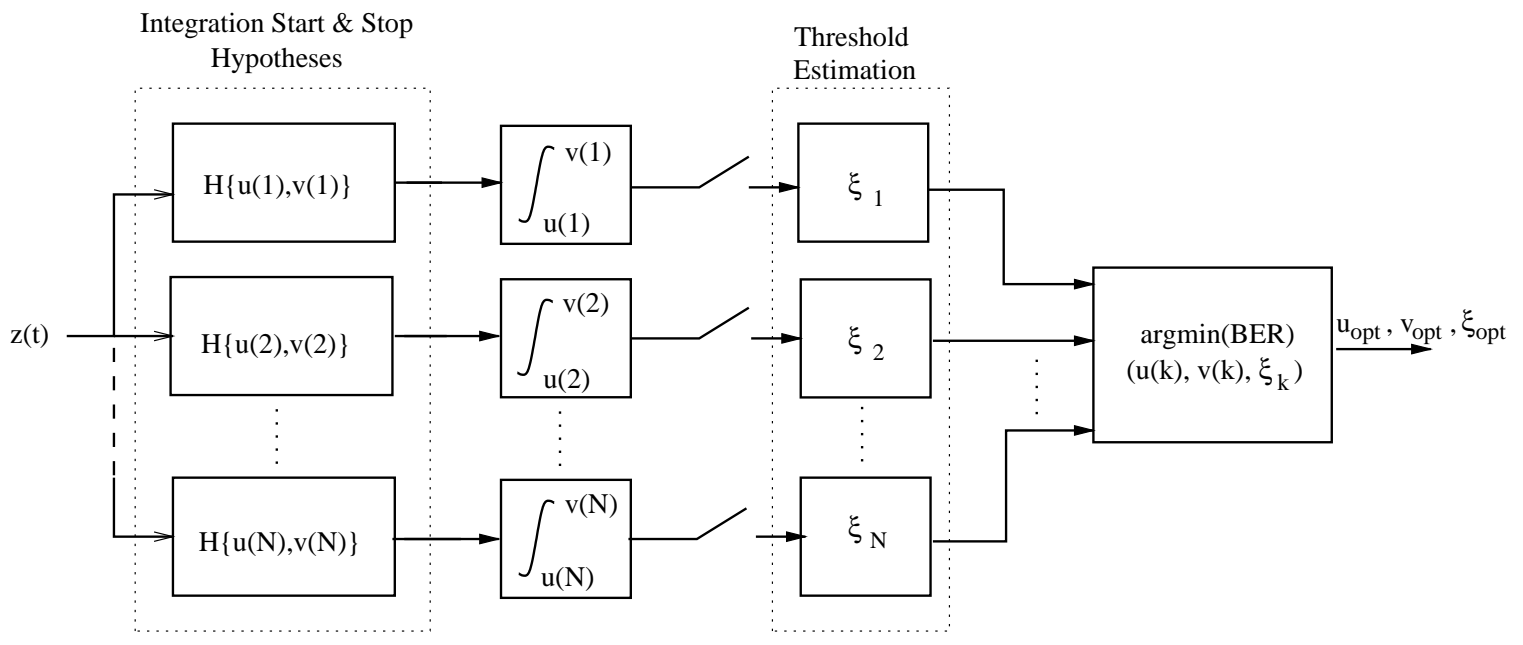

Fig. 2. Block diagram for the proposed joint parameter estimation for energy detector receivers.

\section{SySTEM MODEL}

Let the impulse radio (IR) based UWB signal received for bit $i$ in a multipath environment be represented as

$$
r_{i}(t)=\sum_{l=1}^{L} \gamma_{l} b_{i} \omega_{l}\left(t-\tau_{l}-i T_{s}\right)+n(t)
$$

where $L$ is the number of multipath components arriving at the receiver, $l$ is tap index, $b_{i}$ is the $i$ th transmitted bit with OOK modulation, $\omega_{l}(t)$ is the received pulse shape for the $l$ th path, $\gamma_{l}$ and $\tau_{l}$ are the fading coefficient and the delay of the $l$ th multipath component, respectively, and $T_{s}$ is the symbol duration. The additive white Gaussian noise (AWGN) with double-sided noise spectral density $N_{0} / 2$ is denoted by $n(t)$. The received signal is passed through a bandpass filter of bandwidth $B$ to capture the significant portion of signal spectrum while removing out-of-band noise and interference, resulting in $\tilde{r}(t)$. For the sake of simplicity, we consider single pulse per symbol; however, the discussion in the sequel also (generally) applies to multiple pulses per symbol. The following decision statistic is used to make a symbol detection by sensing if there is energy or not within the symbol interval

$$
h_{i}=\int_{T_{i}}|\tilde{r}(t)|^{2} d t \underset{0}{\stackrel{1}{\gtrless}} \xi,
$$

where $T_{i}$ is the integration window defined by synchronization and dump points $(u, v)$, and the symbol decision is performed by comparing $h_{i}$ with a threshold $\xi$. Observing (2), it is seen that optimal (joint) estimation of $(u, v, \xi)$ tuple is of critical importance for the performance of energy detectors, as will be discussed throughout the rest of this paper.

\section{Optimum Joint Parameter Selection}

Wireless communication systems typically require the estimation of channel-related parameters for optimal demodulation of received symbols. Since channel characteristics change in time, the parameter estimation 
has to be tracked and/or repeated every once in a while; how often the parameter estimation has to be repeated depends on the coherence time of the channel. A commonly used model for UWB channels is a block fading channel model [4], where the channel is assumed stationary within a specific block (e.g. for 200 microseconds [5]), and different channel realizations are considered for different blocks. Therefore, the radio channel characteristics vary in the long-term, and they may be assumed stationary in the short-term.

Since the optimal parameters for an energy detector will vary for different channel realizations, a receiver design that optimizes the performance for a particular channel realization is needed. As illustrated in Fig. 1, the parameters can be estimated at the beginning of each block, and then be used for demodulation of the symbols for the rest of the block. The proposed adaptive receiver, which takes into account the changes in the channel, is shown in Fig. 2. In this receiver, the received signal is first amplified, band pass filtered, and squared. Then, different hypotheses for $(u, v)$ are considered, and the corresponding threshold is estimated for each hypothesis. In this section, first, issues related to obtaining the integrator start/stop hypotheses will be discussed. Then, exact and Gaussian approaches for threshold estimation will be presented.

\section{A. Obtaining the $(u, v)$ Hypotheses with Different Sampling Approaches}

When implementing an energy detector, specifying an integration interval that sacrifices the insignificant multipath components in order to decrease the collected noise energy will improve the performance.For a better performance it is also required that the receiver synchronizes with the starting point of the multipath energy. Therefore, the optimal interval, which minimizes the BER, can ideally be achieved by a joint and adaptive determination of the starting point and duration of integration.

Let $u(k)$ and $v(k)$ denote the starting and dump points of the $k$ th hypothesis, respectively. Granularity of the $(u(k), v(k))$ pair depends on the sampling rate, and they may be obtained using different architectures. Below we present three convenient ways of obtaining the start/stop points for the multiple hypothesis:

1) Multiple parallel integrator branches: Each branch has a different time constant and hence a different length of integration interval. Integration starting points are adjusted using delay elements. The integrator outputs are sampled at a symbol-spaced rate, and the effective granularity is $T_{s} / N$, where $N$ is the number of integrators. The disadvantage of this approach is the large number of integrators that may be required.

2) Single integrator with high sampling rate: The high-rate sampling at a rate $T_{s} / N$ enables determining the energy in finer resolution. The starting and stop points are selected by combining these sample energies in such a way to yield the optimum total energy. The drawback compared with other options is the requirement of a high speed analog to digital converter (ADC). Multiple parallel ADCs may also be considered to increase the sampling rate.

3) Single integrator employing training sequences: Training sequences longer than usual enable testing different integration intervals in a sequential manner. Symbol-rate sampling of the integrator is sufficient. However, since large number of training symbols are required to increase the sampling rate, the coherence time of the channel should be sufficiently long. On the other hand, since symbol-rate sampling will be used in the symbol demodulation anyway, this is the least complex implementation of the receiver.

Note that increasing the rate at which the output of the integrator is sampled, in effect, increases the 'integration time resolution' of the receiver and enhances the likelihood of obtaining a lower BER. However, this comes at the expense of additional hardware complexity. On the other hand, high sampling rates are required only when estimating the integration start/stop times, and symbol-spaced sampling is sufficient during symbol detection. Nevertheless, we assume in the sequel that using one of the above approaches, the integration start/stop hypothesis become available to the receiver.

A sub-optimal solution, where the initial point of the received signal is taken as the common starting point for all possible integration durations, yields very close performance to the optimal case, when the power delay profile (PDP) of the channel realization is exponentially decaying. For example, the channel model CM1 in [4] reflects such a minimum phase scenario where single synchronization point performs as well. For dispersive channels (such as CM4) however, there will be some performance degradation. 


\section{B. Threshold Selection Using Exact and Gaussian Analysis}

The exact optimal threshold $\xi_{k}^{(E)}$ can be calculated using the centralized and non-centralized Chi-square distributions, corresponding to bits 0 and 1 , respectively, and where $k$ denotes the hypothesis number. However, this requires a search over possible threshold values in order to find the one that minimizes the BER, or, high signal to noise ratio (SNR) assumption in order to use asymptotic approximation of the Bessel function (which still yields a threshold estimate based on tabulated data) [6]. Relying on the fact that the normalized threshold for practical SNR values falls in between 0.25 and 0.5 [3], in order to decrease the computational complexity, we consider here a serial search for $\xi_{k}^{(E)}$ in the range $\left(M N_{0}+0.5 E_{b}, M N_{0}+E_{b}\right)$, where $M$ is the degree of freedom (DOF) defined by $2 M=2 B T_{i}+1$, and $E_{b}$ is the average energy of bits 0 and 1 , bit 1 having an energy of $2 E_{b}$.

By approximating the Chi-square distributions with Gaussian distributions (which becomes more valid for large DOF), the threshold estimates $\xi_{k}^{(G)}$ can be obtained (as an approximation to $\xi_{k}^{(E)}$ ). Even though these estimates are suboptimal, they can be obtained easily, without requiring any search over possible threshold values. Let the means and variances of the Chi-square distributions for bits 0 and 1 be given by $\mu_{0, k}, \sigma_{0, k}^{2}, \mu_{1, k}$, and $\sigma_{1, k}^{2}$, respectively, where

$$
\begin{aligned}
\mu_{0, k} & =M N_{0} \\
\sigma_{0, k}^{2} & =M N_{0}^{2} \\
\mu_{1, k} & =M N_{0}+2 E_{b} \\
\sigma_{1, k}^{2} & =M N_{0}^{2}+4 E_{b} N_{0} .
\end{aligned}
$$

The threshold estimate using the Gaussian approximation is located at the intersection of the two Gaussian distributions, which can be evaluated from

$$
\frac{\exp \left(-\frac{\left(\xi_{k}^{(G)}-\mu_{0, k}\right)^{2}}{2 \sigma_{0, k}^{2}}\right)}{\sqrt{2 \pi \sigma_{0, k}^{2}}}=\frac{\exp \left(-\frac{\left(\mu_{1, k}-\xi_{k}^{(G)}\right)^{2}}{2 \sigma_{1, k}^{2}}\right)}{\sqrt{2 \pi \sigma_{1, k}^{2}}} .
$$

Taking the natural logarithm of both sides and rearranging the terms, one obtains

$$
C_{1}\left(\xi_{k}^{(G)}\right)^{2}+C_{2} \xi_{k}^{(G)}+C_{3}=0
$$

where the coefficients are given by

$$
\begin{aligned}
& C_{1}=\sigma_{1, k}^{2}-\sigma_{0, k}^{2} \\
& C_{2}=-2\left(\mu_{0, k} \sigma_{1, k}^{2}-\mu_{1, k} \sigma_{0, k}^{2}\right), \\
& C_{3}=\sigma_{1, k}^{2} \mu_{0, k}^{2}-\sigma_{0, k}^{2} \mu_{1, k}^{2}-2 \sigma_{0, k}^{2} \sigma_{1, k}^{2} \ln \left(\frac{\sigma_{1, k}}{\sigma_{0, k}}\right),
\end{aligned}
$$

with (8) being a second order polynomial equation that can be easily solved for $\xi_{k}^{(G)}$ (only one of the roots is appropriate) yielding

$$
\xi_{\text {opt }}=\frac{-C_{2}+\sqrt{C_{2}^{2}-4 C_{1} C_{3}}}{2 C_{1}} .
$$

As an alternative to using frequent training symbols, the threshold can be updated (tracked) in a decisiondirected manner once it is initially estimated in a similar way to a data-aided channel estimation [7]. 


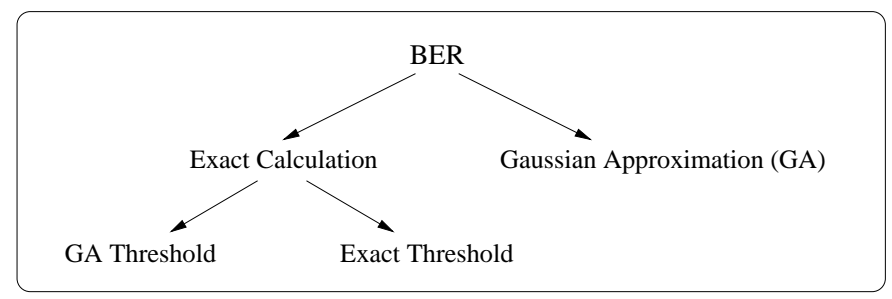

Fig. 3. Three different approaches for performance evaluation.

\section{BER PERformance EVAluation}

We consider three different approaches for evaluating the BER of the energy detector receivers as summarized in Fig. 3. Due to the square-law device used in the receiver, the decision statistics in an energy detector have a Chi-square distribution. First, we consider the exact statistics, and evaluate the BER expressions as available in the literature. However, we consider the threshold using both the exact approach (using a search over possible threshold values) and the Gaussian approximation (using analytical expressions obtained in previous sections). Later, we consider the BER evaluation using the Gaussian approximation of the Chi-square statistics.

\section{A. Exact BER Performances}

When the exact Chi-square statistics of the received signal are considered, the BER observed for each hypothesis when using a serial search or a Gaussian approximation for threshold estimation are denoted by $P_{b}^{(E)}\left(k, \xi_{k}^{(E)}\right)$ and $P_{b}^{(E)}\left(k, \xi_{k}^{(G)}\right)$, respectively. Using the exact expressions, the BERs employing either threshold are given by

$$
\begin{aligned}
P_{b}^{(E)}\left(k, \xi_{k}\right) & =P_{k, \xi_{k}}^{(E)}(0 \mid 1)+P_{k, \xi_{k}}^{(E)}(1 \mid 0), \\
P_{k, \xi_{k}}^{(E)}(0 \mid 1) & =0.5-0.5 \mathcal{Q}_{M}\left(\sqrt{\frac{4 E_{b}}{N_{0}}}, \sqrt{\frac{2 \xi_{k}}{N_{0}}}\right), \\
P_{k, \xi_{k}}^{(E)}(1 \mid 0) & =\frac{e^{-\frac{\xi_{k}}{N_{0}}}}{2} \sum_{u=1}^{\lfloor\rfloor\rfloor} \frac{\left(\xi_{k} / N_{0}\right)^{M-u}}{\Gamma(M-u+1)},
\end{aligned}
$$

where $\mathcal{Q}_{M}$ is the generalized Marcum- $\mathcal{Q}$ function of order $M$, and $\Gamma(x)$ is the Gamma function equal to $(x-1)$ ! for $x$ integer. The optimum integrator parameters are the ones that minimize the BER, i.e.

$$
\left(u_{o p t}, v_{o p t}, \xi_{o p t}\right)=\underset{u(k), v(k), \xi_{k}}{\operatorname{argmin}}\left(P_{b}^{(E)}\left(k, \xi_{k}\right)\right) .
$$

As an alternative to minimizing the BER, one may consider to maximize the SNR (which has less complexity since no BER expressions are evaluated). However, the definition of SNR is critical in energy detectors. One may define the SNR to be the ratio of the square of the mean-shift due to the existence of signal to the output noise variance when signal is present [8], which is expressed as

$$
\begin{aligned}
\mathrm{SNR} & =\frac{\left(\mu_{1, k}-\mu_{0, k}\right)^{2}}{\sigma_{1, k}^{2}}, \\
& =\frac{4 E_{b}^{2}}{M N_{0}^{2}+4 E_{b} N_{0}},
\end{aligned}
$$

and the parameters that maximize (17) can be selected. However, note that (17) does not account for the noise statistics when signal is not present, and thus does not capture the whole picture. This is as opposed to a coherent system, where noise statistics corresponding to both bit- 0 and bit- 1 are identical, and maximization of the SNR implies the minimization of the BER. 


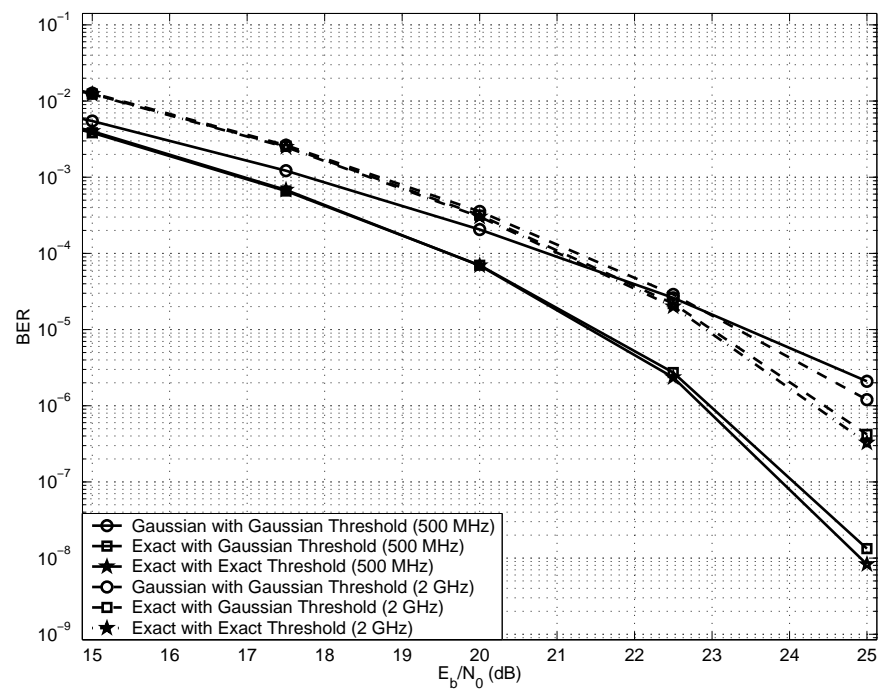

Fig. 4. Bit error rate vs. $E_{b} / N_{0}$ for $\mathrm{CM} 1(\mathrm{BW}=500 \mathrm{MHz}$ and $2 \mathrm{GHz}$ cases) for both Gaussian approximated and exact threshold estimates.

\section{B. BER Using the Gaussian Approximation}

For theoretical purposes, an approximate BER formulation that gives a feasible estimate for $P_{b}\left(k, \xi_{k}\right)$ is given by

$$
P_{b}^{(G)}\left(k, \xi_{k}^{(G)}\right)=\frac{1}{2} Q\left(\frac{\xi_{k}^{(G)}-\mu_{0, k}}{\sigma_{0, k}^{2}}\right)+\frac{1}{2} Q\left(\frac{\mu_{1, k}-\xi_{k}^{(G)}}{\sigma_{1, k}^{2}}\right) .
$$

Since the Chi-square statistics can be approximated with a Gaussian for large degree of freedoms, the above expression is expected to approximate the BER at large bandwidths, or large integration intervals. It is also valid for systems that use large number of pulses per symbol ${ }^{1}$.

\section{NUMERICAL RESULTS}

Computer simulations are done to analyze the performances of the proposed approaches using the channel models in [4]. To be more specific, in these simulations the energies corresponding to the different channel realizations and parameter sets are evaluated, and used in the BER expressions.

In Fig. 4, the BERs obtained using the three different performance analysis approaches (shown in Fig. 3) are compared for $B=0.5 \mathrm{GHz}$ and $B=2 \mathrm{GHz}$. While the Gaussian approximation fails to yield close results to the exact expressions for $B=0.5 \mathrm{GHz}$, we see that the approximation error decreases as the bandwidth increases. On the other hand, for both bandwidths practical estimation of the threshold using the Gaussian approximation yields very close results with the exact threshold (which has to be calculated after a serial search). Hence, the GA threshold can be employed to decrease the computational complexity.

Another observation is that the optimum integration interval changes substantially for different channel models, implying the fact that significant gains can be obtained for a mobile device when the integration interval is adaptively determined. Both the BER minimization and SNR maximization approaches are employed to find the optimum integration interval. The results are shown in Fig. 5 and in Fig. 6, respectively. Although the resulting curves have a similar behaviour, the optimum integration intervals determined by the SNR maximization approach turn out to yield higher BERs than the ones found with the BER minimization. Therefore, we conclude that minimizing the BER is favorable to maximizing SNR despite its computational complexity.

\footnotetext{
${ }^{1}$ Note that if more then one pulse is used per symbol, and the pulses are combined non-coherently, the number of pulses can be folded into the integration interval, implying that the decision statistics approach to a Gaussian distribution
} 


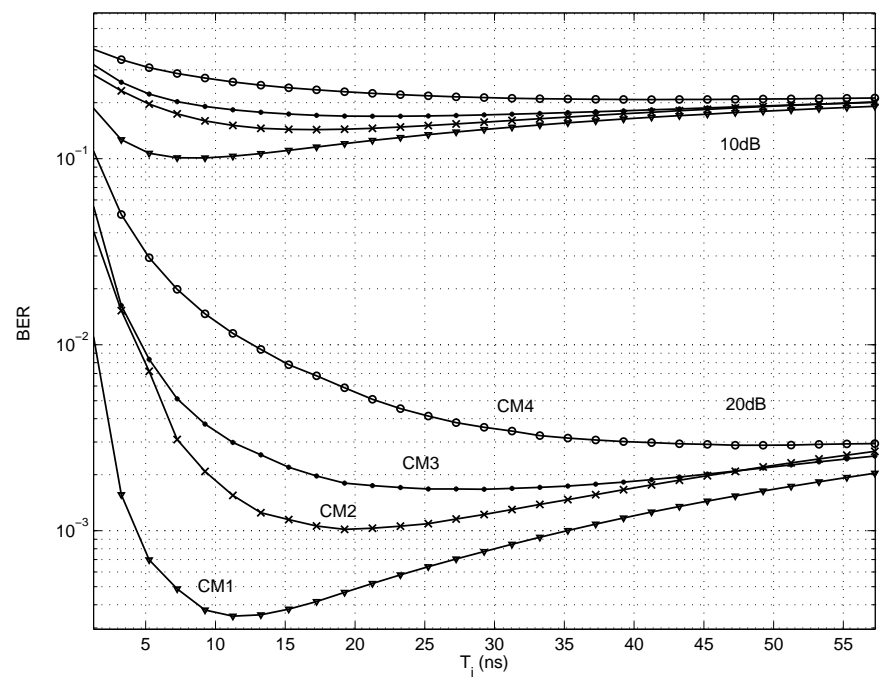

Fig. 5. BER vs. Integration interval for different channel models (at $E_{b} / N_{0}=10 \mathrm{~dB}$ and $20 \mathrm{~dB}$ ).

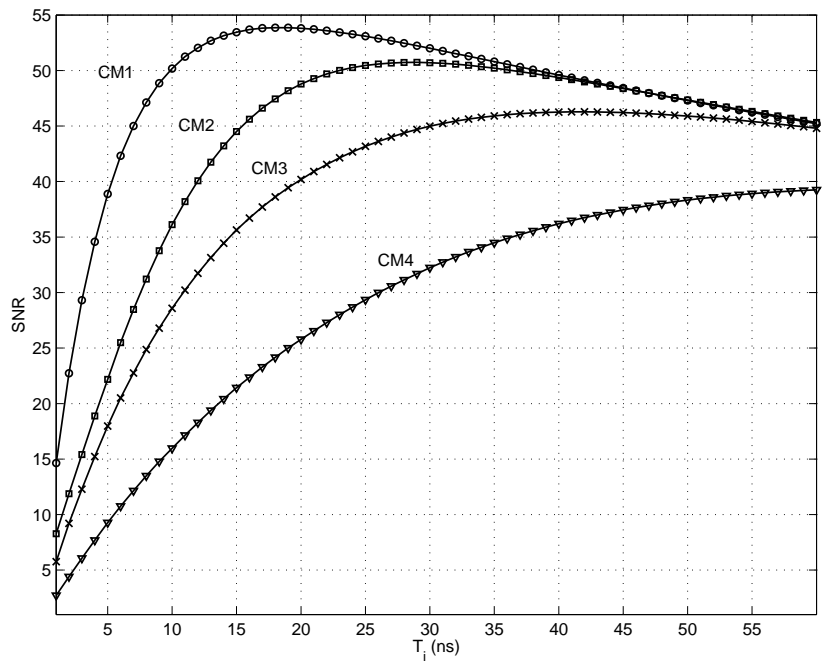

Fig. 6. SNR vs. Integration interval for different channel models (at $E_{b} / N_{0}=20 \mathrm{~dB}$ ).

In Fig. 7, the variation of the optimal integration interval with respect to $E_{b} / N_{0}$ is plotted for different channel models. It is observed that the line-of-sight (LOS) component of CM1 yields a parallel variation with CM2. On the other hand, CM3 and CM4 also exhibit a parallel behaviour and they have larger optimal integration values (and slopes) due to the more spread distribution of their multipath components over time.

In Fig. 8, we compare the BER performances of a non-adaptive receiver and the proposed receiver. The non-adaptive receiver is assumed to have a fixed integration interval of $20 \mathrm{~ns}$, which is a reasonable duration considering the optimum values for different channel models given in Fig. 7. The resultant BER curves are presented for CM1 and CM4. The performance of the proposed receiver is better than the non-adaptive receiver with an appropriately selected fixed integration interval by approximately $1 \mathrm{~dB}$. In the same figure, the synchronization effect is also illustrated. Synchronization is achieved by having the receiver synchronize itself with the starting point of the optimum integration interval rather than the initial multipath component. 
It is seen that the effect of synchronization is negligible for CM1 and very slight for CM4.

In the previous simulations, perfect parameter estimates for (3)-(6) were considered. Another analysis investigates how the number of training symbols affects the parameter estimation and, as a result, the BER. This is an analytical examination rather than a simulation, and therefore, practical channel realizations are not considered. In Fig. 9, the BER vs. number of training symbols curves are plotted at different $E_{b} / N_{0}$ values. These results are obtained by taking samples from the centralized and non-centralized Chi-square distributions of bit- 0 and bit-1, respectively. Each sample corresponds to a training symbol transmitted. Obviously, taking more samples yields a better estimate for the symbol energy. A significant conclusion that can be drawn from this figure is that as $E_{b} / N_{0}$ increases, the number of training symbols required to converge to the optimum BER increases as well. The reason for this fact is that as the signal energy rises, the probability density function for bit-1 becomes broader, and hence, more samples are required for a more accurate estimation. The theoretical optimum BERs are also indicated on the figure. Note that these BERs are different from the ones shown in Fig. 5. This is because in this analysis, the entire symbol energy is considered rather than only the energy confined to the integration interval.

\section{CONCLUSION}

In this paper, the need for the joint adaptation of the integration interval, optimal threshold, and the synchronization point (for certain channels) is demonstrated. Even though the Gaussian approximation does not lead to a correct BER evaluation, it closely approximates the exact threshold values. SNR maximization is shown to yield suboptimal parameter estimates compared to BER minimization.

\section{REFERENCES}

[1] M. Weisenhorn and W. Hirt, "Robust noncoherent receiver exploiting UWB channel properties," in Proc. IEEE Ultrawideband Syst. Technol. (UWBST), Kyoto, Japan, May 2004, pp. 156-160.

[2] A. Rabbachin and I. Oppermann, "Synchronization analysis for UWB systems with a low-complexity energy collection receiver," in Proc. IEEE Ultrawideband Syst. Technol. (UWBST), Kyoto, Japan, May 2004, pp. 288-292.

[3] P. A. Humblet and M. Azizoglu, "On the bit error rate of lightwave systems with optical amplifiers," J. of Lightwave Technology, vol. 9, no. 11, pp. 1576-1582, Nov. 1991.

[4] J. Foerster, "IEEE P802.15 working group for wireless personal area networks (WPANs), channel modeling sub-committee report final," Mar. 2003. [Online]. Available: http://www.ieee802.org/15/pub/2003/Mar03/

[5] B. Mielczarek, M. Wessman, and A. Svensson, "Performance of coherent uwb rake receivers with channel estimators," in Proc. IEEE Vehic. Technol. Conf., vol. 3, Orlando, FL, Oct. 2003, pp. 1880-1884.

[6] S. Paquelet, L. M. Aubert, and B. Uguen, "An impulse radio asynchronous transceiver for high data rates," in Proc. IEEE Ultrawideband Syst. Technol. (UWBST), Kyoto, Japan, May 2004, pp. 1-5.

[7] V. Lottici, A. D'Andrea, and U. Mengali, "Channel estimation for ultra-wideband communications," IEEE J. Select. Areas Commun., vol. 20, no. 9, pp. 1638-1645, Dec. 2002.

[8] N. F. Krasner, "Efficient search methods using energy detectors-maximum probability of detection," IEEE J. Select. Areas Commun., vol. 4, no. 2, pp. 273-279, March 1986. 


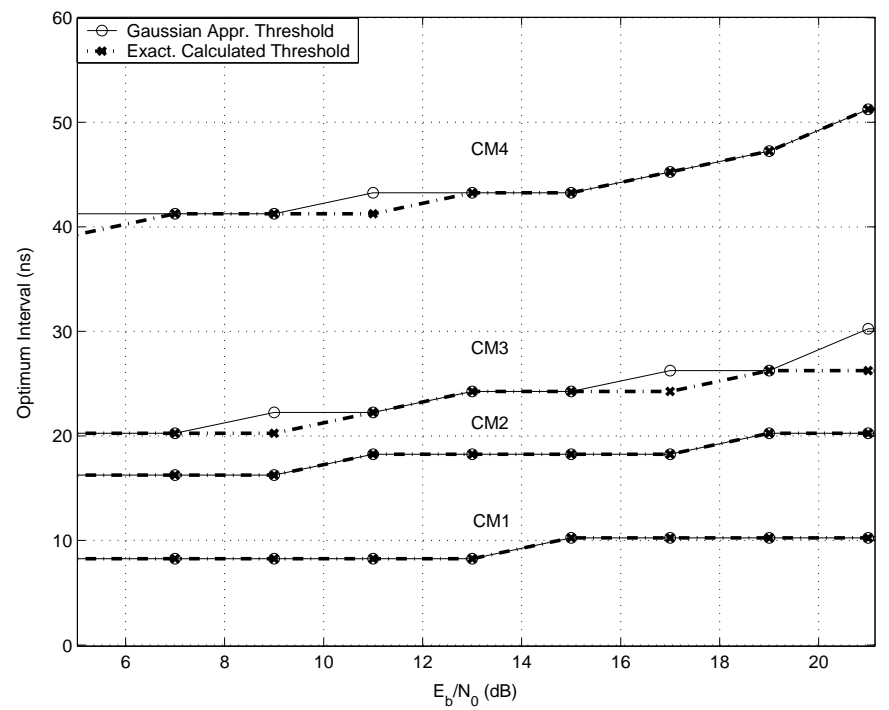

Fig. 7. Optimum integration interval vs. $E_{b} / N_{0}$ for different channel models.

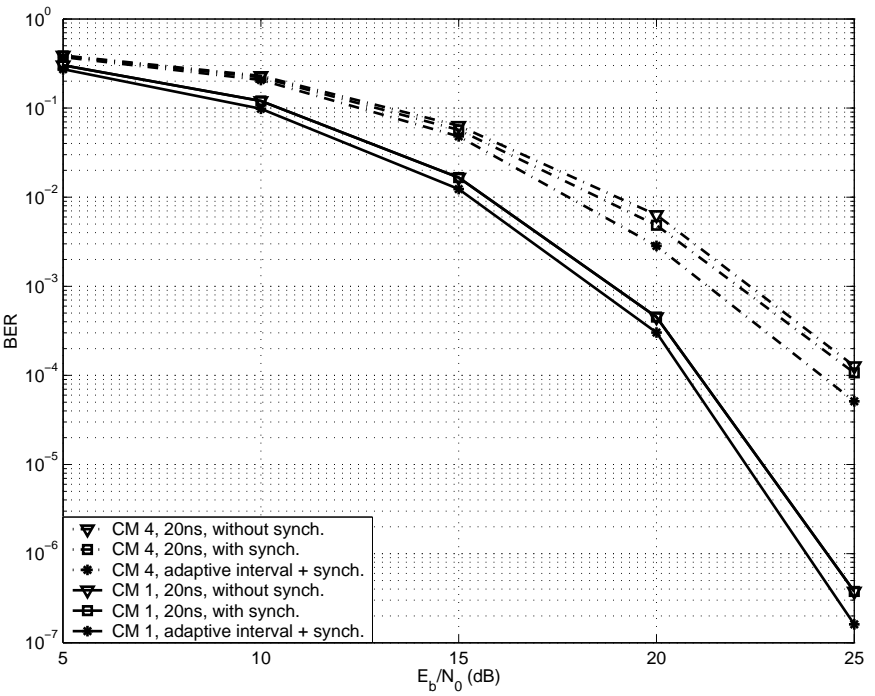

Fig. 8. BER vs. $E_{b} / N_{0}$ for fixed integration intervals, adaptive integration interval, and adaptive synchronization point. 


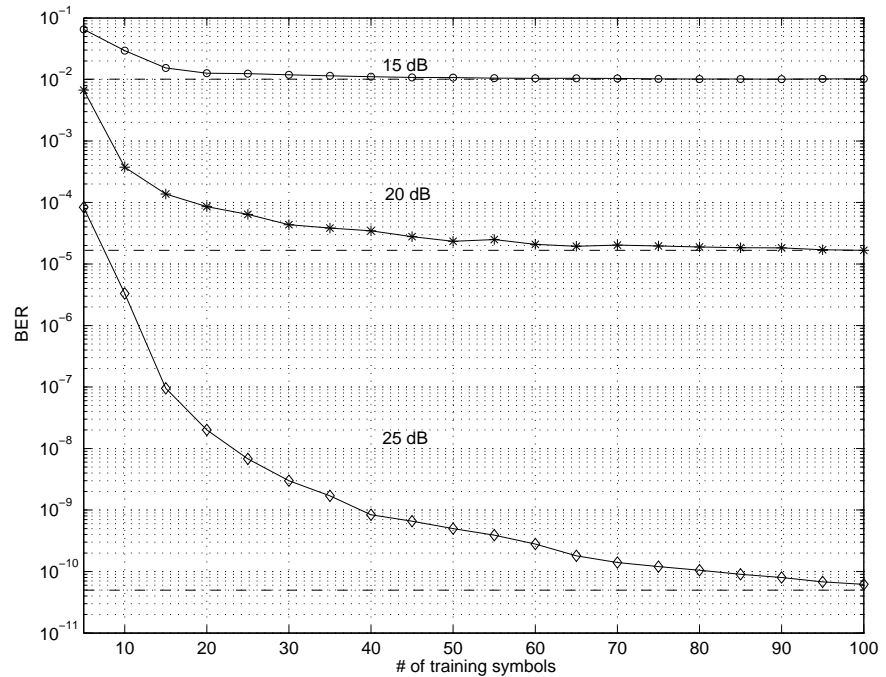

Fig. 9. Analytical results regarding BER vs. number of training symbols (at $E_{b} / N_{0}=15 \mathrm{~dB}, 20 \mathrm{~dB}$, and $25 \mathrm{~dB}$ ). 